\title{
Resource Allocation for Physical Layer Security in 5G Full- Duplex Communication System: an Iterative Matching Approach
}

\author{
Song Li \\ School of Information and Control \\ Engineering \\ China University of Mining and \\ Technology, \\ Xuzhou, China, 221116 \\ lisong@cumt.edu.cn \\ Qi Cao \\ China University of Mining and \\ Technology, \\ Xuzhou, China, 221116 \\ qcao@cumt.edu.cn
}

\author{
Yanjing Sun \\ School of Information and Control \\ Engineering \\ China University of Mining and \\ Technology, \\ Xuzhou, China, 221116 \\ yjsun@cumt.edu.cn \\ Shuo Li \\ School of Information and Control \\ Engineering \\ China University of Mining and \\ Technology, \\ Xuzhou, China, 221116 \\ lishuosd@163.com
}

\begin{abstract}
Full-duplex is a promising technology in the next generation wireless communication system to improve the spectrum efficiency by receiving and transmitting simultaneously in the same channel. In this paper, we investigate resource allocation problem aiming to enhance physical layer security of full-duplex cellular system with half-duplex (HD) users and full-duplex (FD) base station. The full-duplex resource allocation is formulated as 3-dimentional one-to-one matching problem and an iterative Hungarian method (IHM) is proposed to solve the matching problem. Simulation results show that the IHM can achieve optimum solution of the integer optimization problem while maintaining a rather low computational complexity.
\end{abstract}

\section{KEYWORDS}

full-duplex, resource allocation, physical layer security, iterative Hungarian method

\section{INTRODUCTION}

In next generation wireless communication (5G), various techniques have been proposed to improve the spectrum

Permission to make digital or hard copies of all or part of this work for personal or classroom use is granted without fee provided that copies are not made or distributed for profit or commercial advantage and that copies bear this notice and the full citation on the first page. To copy otherwise, to republish, to post on servers or to redistribute to lists, requires prior specific permission and/or a fee.

Mobimedia 2017, July 13-14, Chongqing, People's Republic of China

Copyright () 2017 EAI 978-1-63190-156-0 mobile phones and emerging mobile services, such as mobile TV, online games and internet of vehicles. In-band full-duplex communication enables a transceiver receiving and transmitting signals simultaneously using the same spectrum resource, to improve the spectrum efficiency, and thus become a promising technology in 5G[1]. Full-duplex technique (FD) can be implemented on the condition that the self-interference can be effectively suppressed by self-interference cancellation method, such as antenna isolation and active cancellation[2].

In various mobile applications, such as mobile online shopping and mobile health, security in communication becomes a more and more important issue to users. The broadcast nature of wireless channel brings much difficulty to the security issue of wireless communication. Physical layer security technology provides another way in physical layer to enhance security for wireless terminal from information theoretical perspective, besides encryption and authentication in higher layer[3][4]. The theoretical approach of physical layer security is to improve the capacity of legitimate user and while reducing the capacity of eavesdropper, by beamforming, power allocation, relay selection, et al. Recently, physical layer security technology has been widely investigated in various scenarios, including relaying[5], nor-orthogonal multiple access[6] and device-to-device communications[7].

The physical layer security problem for full-duplex communications has also been investigated [8][9]. In [8], in a cellular system with one full-duplex base station, one uplink user, one downlink user and one eavesdropper, the secret transmit rate maximization problem with secret receive rate constraint is considered through joint information and jamming beamforming. In [9], the power minimization problem is investigated while 
guaranteeing physical layer security of uplink user and downlink user.

The existing researches on physical layer security for full-duplex cellular system mainly focus on single user pair scenario (one uplink user and one downlink user). To the best of our knowledge, the multiple user pairs scenario has not been concerned. In this paper, we investigated the resource allocation problem in full-duplex cellular system with multiple users, aiming to maximize total security capacity of uplink users and downlink users. The optimization problem is formulated as a 3dimentional matching problem and an iterative Hungarian method is proposed to solve 3-dimentional matching problem with a lower complexity. Simulation results show that the proposed method can nearly approach the performance of exhaustive searching while maintain a much lower complexity.

\section{SYSTEM MODEL AND PROBLEM FORMULATION}

\subsection{System Model}

We consider a single cell with one FD base station (BS), multiple HD uplink users, multiple HD downlink users as illustrated in Fig.1. FD BS can serve one uplink user and one downlink users at one channel $(\mathrm{CH})$ simultaneously. One eavesdropper attempts to wiretap the signals transmitted by uplink users to BS and transmitted by BS to downlink users. We assume that each user, including legitimate user and eavesdropper, is equipped with one antenna and BS is equipped with a receiving antenna and transmitting antenna. The set of uplink users and downlink users is denoted as $\mathcal{T}=\left\{T U_{1}, T U_{2}, \ldots T U_{M}\right\}$ and $\mathcal{R}=\left\{R U_{1}, R U_{2}, \ldots\right.$, $\left.R U_{N}\right\}$. The set of $\mathrm{CHs}$ is denoted as $\mathcal{C}=\left\{\mathrm{CH}_{1}, \mathrm{CH}_{2}, \ldots C \mathrm{H}_{K}\right\}$. We assume that the number of $\mathrm{CH}$ is sufficient to support all the downlink users and uplink users, which can be expressed by $K \geq \max \{M, N\}$.

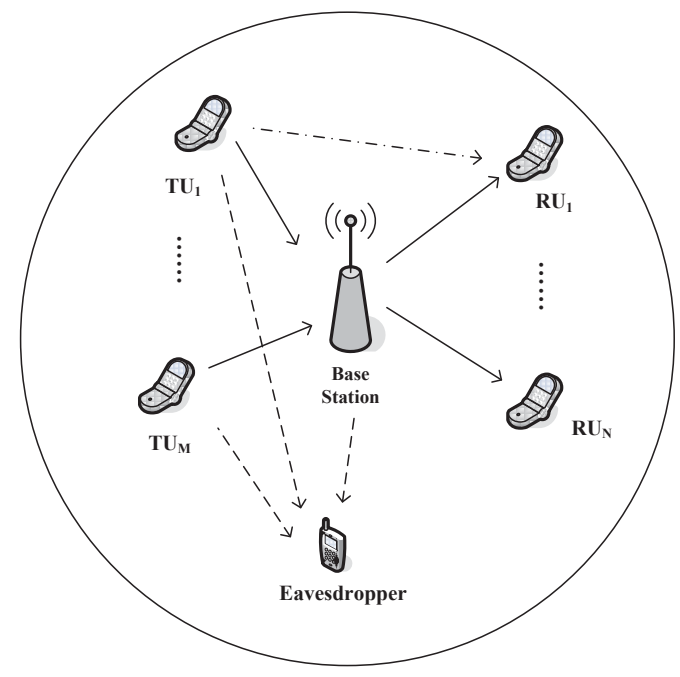

Figure 1 System model of full-duplex cellular system with one eavesdropper
Denote $h_{i, b, k}$ and $h_{b, j, k}$ as the channel gain between $\mathrm{TU}_{i}$ and BS on $\mathrm{CH}_{k}$, and between BS and $\mathrm{RU}_{j}$ on $\mathrm{CH}_{k}$ respectively. Denote $g_{b, k}$ and $g_{i, j, k}$ as equivalent self-interference channel gain after interference cancellation and interference channel gain between $\mathrm{TU}_{i}$ and $\mathrm{RU}_{j}$ respectively. Denote $h_{i, e, k}$ and $h_{b, e, k}$ as channel gain between $\mathrm{TU}_{i}$ and eavesdropper and channel gain between $\mathrm{BS}$ and eavesdropper respectively.

The capacity of uplink channel and downlink on $\mathrm{CH}_{k}$ can be derived according Shannon's theory respectively as:

$$
\begin{aligned}
C_{i, b, k}^{s} & =\sum_{i=1}^{M} \alpha_{i, k} W \log \left(1+\frac{P^{u} h_{i, b, k}}{\sum_{j=1}^{N} \beta_{j, k} P^{d} g_{b, k}+\sigma_{n}}\right) \\
C_{b, j, k}^{s} & =\sum_{j=1}^{N} \beta_{j, k} W \log \left(1+\frac{P^{d} h_{b, j, k}}{\sum_{i=1}^{M} \alpha_{i, k} P^{u} g_{i, j, k}+\sigma_{n}}\right)
\end{aligned}
$$

where $W$ is channel bandwidth of each $\mathrm{CH}, \sigma_{n}$ denote the variance of noise on each user. $\alpha_{i, k}$ and $\beta_{j, k}$ denote allocation index of uplink user and downlink user, respectively. $\alpha_{i, k}=1$ means TUi transmitting signal on $\mathrm{CH}_{\mathrm{k}}$, while $\alpha_{\mathrm{i}, \mathrm{k}}=0$ means TU do not transmits signal on $\mathrm{CH}_{k}$. Likewise, $\beta_{j, k}=1$ means $\mathrm{RU}_{\mathrm{j}}$ is allocated on $\mathrm{CH}_{k}$, while $\beta_{j, k}=0$ means $\mathrm{RU}_{j}$ is not allocated on $\mathrm{CH}_{k}$. One $\mathrm{CH}$ can allocated to no more than one TU and one RU, which can be referred as $\sum_{i=1}^{M} \alpha_{i, k} \leq 1$ and $\sum_{j=1}^{N} \beta_{j, k} \leq 1$. Also, in order to guarantee fairness among users, we assume that one TU can only be allocated one $\mathrm{CH}$. The same assumption is hold for RU. Note that a $\mathrm{CH}$ does not always serve a uplink user and downlink user simultaneously. There are other two cases in which a $\mathrm{CH}$ serves a uplink user or a downlink user only and BS operates in half duplex mode in these cases.

Assume that the eavesdropper can wiretap the transmitted signal on every $\mathrm{CH}$. The signal that eavesdropper receives experiences a MISO channel. The capacity of this MISO channel can be referred to as the information that eavesdropper can wiretaps, which can be formulated as

$$
\begin{gathered}
C_{i, e, k}^{s}=\sum_{i=1}^{M} \alpha_{i, k} W \log \left(1+\frac{P^{u} h_{i, e, k}}{\sum_{j=1}^{N} \beta_{j, k} P^{d} h_{b, e, k}+\sigma_{n}}\right) \\
C_{b, e, k}^{s}=\sum_{j=1}^{N} \beta_{j, k} W \log \left(1+\frac{P^{d} h_{b, e, k}}{\sum_{i=1}^{M} \alpha_{i, k} P^{u} h_{i, e, k}+\sigma_{n}}\right)
\end{gathered}
$$

When $\mathrm{TU}_{i}$ and $\mathrm{RU}_{j}$ are served by $\mathrm{BS}$ on $\mathrm{CH}_{k}$, the security capacity of TU and RU on $\mathrm{CH}_{k}$ can be respectively expressed as

$$
\begin{aligned}
& C_{i, k}^{e}=\max \left\{0, C_{i, b, k}-C_{i, e, k}^{s}\right\}, \quad 1 \leq i \leq M, 1 \leq k \leq K \\
& C_{j, k}^{e}=\max \left\{0, C_{b, j, k}-C_{b, e, k}^{s}\right\}, 1 \leq j \leq N, 1 \leq k \leq K
\end{aligned}
$$

Where $C_{i, k}$ and $C_{j, k}$ are channel capacity of uplink channel and downlink channel on $\mathrm{CH}_{k}$, respectively. $C_{i, j, k}^{s}$ is the capacity of wiretap channel. Then we denote the security capacity on $\mathrm{CH}_{k}$ as the sum of security capacity of TU and $\mathrm{RU}$ on $\mathrm{CH}_{k}$ : 


$$
C_{k}^{e}=\sum_{i=1}^{M} C_{i, k}^{e}+\sum_{j=1}^{N} C_{j, k}^{e}, \quad 1 \leq i \leq M, 1 \leq j \leq N, 1 \leq k \leq K
$$

\subsection{Problem Formulation}

In this paper, we investigated the resource allocation problem of both uplink users and downlink users to maximize the total security capacity. The optimization problem can be formulated as

$$
\begin{aligned}
& \max _{\alpha_{i, k}, \beta_{j, k}} \sum_{k=1}^{K} C_{k}^{e} \\
& \text { s.t. } \sum_{i=1}^{M} \alpha_{i, k} \leq 1, \quad k=\{1,2, \ldots, K\} \\
& \sum_{j=1}^{N} \beta_{j, k} \leq 1 \quad k=\{1,2, \ldots, K\} \\
& \sum_{k=1}^{K} \alpha_{i, k} \leq 1 \quad i=\{1,2 \ldots, M\} \\
& \sum_{k=1}^{K} \beta_{j, k} \leq 1 \quad j=\{1,2 \ldots, N\}
\end{aligned}
$$

The joint resource allocation problem for RU and TU is a $0-1$ optimization problem, which has been proven to be NP-hard in [10]. Exhaustive search requires $2^{M N K}$ options. It is impossible to find optimal solution in a polynomial time.

Since the optimization of $\alpha_{i, k}$ and $\beta_{j, k}$ are interdependent due to the peer effect between TU and RU allocated on the same $\mathrm{CH}$, we denote a 3-dimentional index $[W]_{K \times K \times K}$ indicating the allocation of TUs and RUs, in which $w_{i, j, k}=1$ when $\mathrm{CH}_{k}$ is allocated to $\mathrm{TU}_{i}$ (a real TU or a virtual) and $\mathrm{RU}_{j}$ (a real $\mathrm{RU}$ or a virtual RU).

During the allocation, a $\mathrm{CH}$ can be shared by a TU and a RU, or it can be allocated to a TU or a RU exclusively. To establish a unified expression, we introduce the concept of virtual TU and virtual RU. If $\mathrm{CH}_{k}$ allocated to $\mathrm{TU}_{i}$ and a virtual $\mathrm{RU}$, means that $\mathrm{CH}_{k}$ is allocated on TUi only. Similarly, if $\mathrm{CU}_{k}$ allocated to $\mathrm{RU}_{j}$ and a virtual TU, means that $\mathrm{CH}_{\mathrm{k}}$ is allocated on $\mathrm{RU}_{j}$ only. We expand the set of TUs and RUs as as $\underline{\mathcal{I}}=\left\{T U_{1}, T U_{2}, \ldots T U_{M}, T U\right.$ $\left.{ }_{M+1}, \ldots, T U_{K}\right\}$ and $\underline{\mathcal{R}}=\left\{R U_{1}, R U_{2}, \ldots, R U_{N}, R U_{N+1}, \ldots, R U_{K}\right\}$, where $T U$ ${ }_{M+1}, \ldots, T U_{K}$ and $R U_{N+1}, \ldots, R U_{K}$ are $K-M$ virtual TUs and $K-N$ virtual RUs respectively. After adding virtual TUs and virtual RUs, a $\mathrm{CH}$ is always allocated to a TU (a real TU or a virtual) and a RU (a real RU or a virtual RU).

Then we denote a 3-dimentional security capacity matrix as $\left[C^{e}\right]_{K \times K \times K}$, in which the elements $C_{i, j, k}^{e}$ is defined as follows:

$C_{i, j, k}^{e}=\left\{\begin{array}{lr}C_{i, k}^{e}+\left.C_{j, k}^{e}\right|_{\alpha_{i, k}=1, \beta_{j, k}=1, \alpha_{i, k}=0, \beta_{j^{\prime}, k}=0\left(i^{\prime} \neq i, j^{\prime} \neq j\right)} 1 \leq i \leq M, 1 \leq j \leq N \\ \left.C_{i, k}^{e}\right|_{\alpha_{i, k}=1, \alpha_{i, k}=0\left(i^{\prime} \neq i\right), \beta_{j^{\prime}, k}=0} & 1 \leq i \leq M, N+1 \leq j \leq K \\ \left.C_{j, k}^{e}\right|_{\beta_{j, k}=1, \beta_{j^{\prime}, k}=0\left(j^{\prime} \neq j\right), \alpha_{i^{\prime}, k}=0} & M+1 \leq i \leq K, 1 \leq j \leq N \\ 0 & M+1 \leq i \leq K, N+1 \leq j \leq K\end{array}\right.$

(9)
Then the resource allocation problem can be equivalently transformed as:

$$
\begin{aligned}
& \max _{w_{i, j, k}} \sum_{i=1}^{K} \sum_{j=1}^{K} \sum_{k=1}^{K} w_{i, j, k} C_{i, j, k}^{e} \\
& \text { s.t. } \sum_{i=1}^{K} \sum_{j=1}^{K} w_{i, j, k}=1, \quad k=\{1,2, \ldots, K\} \\
& \sum_{i=1}^{K} \sum_{k=1}^{K} w_{i, j, k}=1, \quad k=\{1,2, \ldots, K\} \\
& \sum_{k=1}^{K} \sum_{j=1}^{K} w_{i, j, k}=1, \quad k=\{1,2, \ldots, K\}
\end{aligned}
$$

According to Constraint (10b), a $\mathrm{CH}$ can only be allocated to a TU-RU pair. Also only one $\mathrm{CH}$ can be assigned to a TU (RU) according to (10c) and (10d) respectively.

\section{ITERATIVE HUNGARIAN BASED RESOURCE ALLOCATION}

\subsection{3-Dimentional Matching}

The optimization problem (10) can be formulated as a 3dimentional one-to-one matching problem. A 3-dimential matching problem is defined as follows:

Definition 1: Given three disjoints set $\mathcal{M}, \mathcal{N}$ and $\mathcal{W}$, a $3-$ dimentional one-to-one matching $\mu$ is defined as a mapping from $\mathcal{M} \cup \mathcal{N} \cup \mathcal{W}$ to subsets of $\mathcal{M} \cup \mathcal{N} \cup \mathcal{W}$, such that for $m \in \mathcal{M}$, $\mu(m)=(n, w), n \in \mathcal{N}, w \in \mathcal{W}$, for $n \in \mathcal{N}, \mu(n)=(m, w), m \in \mathcal{M}, w \in$ $\mathcal{W}$, and for $w \in \mathcal{W}, \mu(w)=(m, n), m \in \mathcal{M}, n \in \mathcal{N}$.

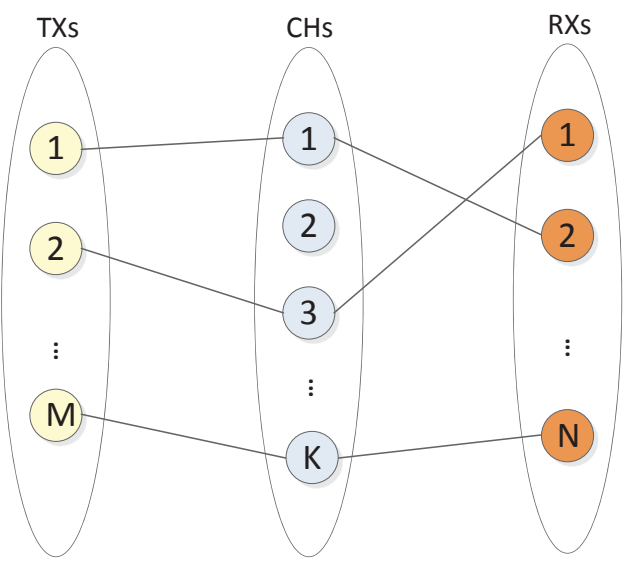

Fig.2 3-dimentional matching problem

Note that unlike 2-dimentional matching problem, also referred as task assignment problem, in which a task should to be assigned to one agent with certain cost, 3-dimentional matching problem can be considered as an enhance task assignment problem in which a task $(\mathrm{CH})$ should be accomplished by two agents (TU and RU) corporately. Different agent pairs have different cost to handle one task. The resource allocation for full- 
duplex communication system is a 3-dimention matching process among three sets $(\mathcal{C}, \mathcal{T}$ and $\mathcal{R})$ and Different matching triple has different cost (security capacity). Our goal is to find the optimal matching to maximize the overall cost.

\subsection{Iterative Hungarian Algorithm}

Since Hungarian algorithm can be used to solve 2-dimentional matching problem (task assignment problem), we proposed an iterative Hungarian algorithm to tackle this 3-dimentional problem.

We set a random allocation matrix $\mathrm{W}_{0}$ as initial allocation, which meet the constraints in (10). Then in each iteration, a 2dimentional matching is implemented between one set and matching pairs of other two sets. According to the matching result of 2-dimentional matching, the 3-dimentional allocation matrix is updated after each iteration until a stable allocation is achieved.

Denote the set of matched RU-CH pairs as $\mathcal{X}_{1}, \mathcal{X}_{1}=\left\{(j, k) \mid w_{i, j, k}=1\right\}$. And we use index $l$ to indicate each RU-CH pair, $1<l<K$ and $l$ is determined as follows: $l=j$. We denote $C_{i, l}^{e}$ as security capacity when $\mathrm{TU}_{i}$ matches the lth $\mathrm{RU}-\mathrm{CH}$ pair. Then the original 3dimentional matching problem is reduced to a 2-dimentional matching problem between $\underline{\mathcal{I}}$ (the set of TUs) and $\mathcal{X}_{1}$ (the set of matched RU-CH pairs). Denote a 2-dimentional index matrix $[\mathrm{X}]_{K_{\times} K}$ to as matching result between TUs and RU-CH pairs, where $x_{i, l}=0$, if $\mathrm{TU}_{i}$ matches the $l$ th $\mathrm{RU}-\mathrm{CH}$ pair, $x_{i, l}=0$, otherwise. The sub-optimization problem can be formulated as:

$$
\begin{aligned}
& \Psi\left(\mathrm{X}^{*}, \mathcal{X}_{1}\right)=\max _{X} \sum_{i=1}^{K} \sum_{l=1}^{K} x_{i, l} C_{i, l}^{e} \\
& \text { s.t. } \sum_{i=1}^{K} x_{i, l} \leq 1, \sum_{l=1}^{K} x_{i, l} \leq 1
\end{aligned}
$$

The optimal solution $\mathrm{X}^{*}$ of 2-dimentional problem (PX.X) can be solved by Hungarian algorithm. By joint considering $\mathrm{X}^{*}$ and $\mathcal{X}_{1}$, we can update the 3 -dimentional allocation matrix $\mathrm{W}_{1}=\left[\mathrm{X}^{*}, \mathcal{X}_{1}\right]$. Denote the set of matched TU-RU pairs as $\mathcal{X}_{2}, \mathcal{X}_{2}=\left\{(i, j) \mid w_{i, j, k}=1\right\}$. And we use index $r$ to indicate each TU-RU pair, $1<\mathrm{r}<K$, and $r$ is determined as follows: $r=m$. We denote $C_{r, k}^{e}$ as security capacity when $\mathrm{CH}_{k}$ matches the $r$ th TU-RU pair. Also we perform a 2dimentional matching between (the set of $\mathrm{CHs}$ ) and $\mathcal{X}_{2}$ (the set of matched TU-RU pairs). Denote a 2-dimentional index matrix $[\mathrm{Y}]_{\mathrm{K}_{\times} \mathrm{K}}$ as matching result between TU-RU pairs and $\mathrm{CHs}$, where $y_{r, k}=1$, if the $r$ th TU-RU pair matches $\mathrm{CH}_{k}, y_{r, k}=0$, otherwise. The sub-optimization problem can be formulated as:

$$
\begin{aligned}
& \Psi\left(Y^{*}, \mathcal{X}_{2}\right)=\max _{Y} \sum_{k=1}^{K} \sum_{r=1}^{K} y_{r, k} C_{r, k}^{e} \\
& \text { s.t. } \sum_{r=1}^{K} y_{r, k} \leq 1, \sum_{k=1}^{K} y_{r, k} \leq 1
\end{aligned}
$$

The optimal solution $Y^{*}$ of 2-dimentional problem (12) can be solved by Hungarian algorithm. Then we can update the 3dimentional allocation matrix $\mathrm{W}_{2}=\left[Y^{*}, \mathcal{X}_{2}\right]$.

Denote the set of matched TU-CH pairs as $\mathcal{X}_{3}, \mathcal{X}_{3}=\left\{(i, k) \mid w_{i, j, k}=1\right\}$. And we use index $d$ to indicate each TU-CH pair, $1<d<K$, and $d$ is determined as follows: $d=m$. We denote $C_{d, j}^{e}$ as security capacity when $\mathrm{RU}_{j}$ matches the $d$ th $\mathrm{TU}-\mathrm{CH}$ pair. Then we perform a 2-dimentional matching between $\underline{\mathcal{R}}$ (the set of RUs) and $\mathcal{X}_{3}$ (the set of matched TU-CH pairs). Denote a 2dimentional index matrix $[Z]_{\mathrm{K}_{\times} \mathrm{K}}$ as matching result between RUs and TU-CH pairs, where $z_{d, j}=1$ if the $d$ th $\mathrm{TU}-\mathrm{CH}$ pair matches $\mathrm{RU}_{\mathrm{j}} ; z_{d, j}=0$, otherwise. The sub-optimization problem can be formulated as:

$$
\begin{aligned}
& \Psi\left(Z^{*}, \mathcal{X}_{3}\right)=\max _{Z} \sum_{j=1}^{K} \sum_{d=1}^{K} z_{d, j} C_{d, j}^{e} \\
& \text { s.t. } \sum_{d=1}^{K} z_{d, j} \leq 1, \sum_{j=1}^{K} z_{d, j} \leq 1
\end{aligned}
$$

The optimal solution $\mathrm{Z}^{*}$ of 2-dimentional problem can be solved by Hungarian algorithm. Then the 3-dimentional allocation matrix can be updated as $\mathrm{W}_{3}=\left[Z^{*}, \mathcal{X}_{3}\right]$.

\begin{tabular}{|c|c|}
\hline \multicolumn{2}{|c|}{ Algorithm 1 Iterative Hungarian Algorithm } \\
\hline \multicolumn{2}{|c|}{ Input: $C^{e}$} \\
\hline \multicolumn{2}{|c|}{ Output: W } \\
\hline 1: & Initialization $\mathrm{W}_{0}$, iter $=0, \mathrm{i}=1$. \\
\hline 2: & while iter $<=$ MAXITER or allocation result remain stable \\
\hline 3: & Matching between TUs and RU-CH pairs \\
\hline \multirow[t]{3}{*}{ 4: } & obtain matched $\mathrm{RU}-\mathrm{CH}$ pairs set $\mathcal{X}_{1}, 2$ \\
\hline & dimentional security capacity $C_{i, l}^{e}$ with ith $\mathrm{TU}$ \\
\hline & and $l$ th RU-CH pair \\
\hline 5: & Solve 2-dimentional matching problem (11) using \\
\hline & Hungarian method and get optimal allocation $\mathrm{X}^{*}$ \\
\hline 6: & Allocation result Update $\mathrm{W}_{\mathrm{i}}=\left[\mathrm{X}^{*}, \mathcal{X}_{1}\right], \mathrm{i}=\mathrm{i}+1$ \\
\hline 7: & Matching between $\mathrm{CH}$ and TU-RU pairs \\
\hline \multirow[t]{3}{*}{ 8: } & obtain matched TU-RU pairs set $\mathcal{X}_{2}, 2$ \\
\hline & dimentional security capacity $C_{r, k}^{e}$ with kth $\mathrm{CH}$ \\
\hline & and $r$ th TU-RU pair \\
\hline 9: & Solve 2-dimentional matching problem (12) using \\
\hline & Hungarian method and get optimal allocation $\mathrm{Y}^{*}$ \\
\hline 10: & Allocation result Update $\mathrm{W}_{\mathrm{i}}=\left[\mathrm{Y}^{*}, \mathcal{X}_{2}\right], \mathrm{i}=\mathrm{i}+1$ \\
\hline 11: & Matching between RU and TU-CH pairs \\
\hline \multirow[t]{3}{*}{ 12: } & obtain matched TU-CH pairs set $\mathcal{X}_{3}, 2-$ \\
\hline & dimentional security capacity $C_{d, j}^{e}$ with jth $\mathrm{RU}$ \\
\hline & and $d$ th TU-CH pair \\
\hline
\end{tabular}

The iteration continues until maximum iteration is achieved or the 3-dimentional allocation matrix remains the same. The 3dimentional allocation matrix is updated as follows $\mathrm{W}_{0} \rightarrow \mathrm{W}_{1} \rightarrow \mathrm{W}_{2} \rightarrow \mathrm{W}_{3} \rightarrow \mathrm{W}_{4} \rightarrow \ldots$ The details of iterative Hungarian algorithm are listed in Algorithm 1. 

Hungarian method and get optimal allocation $\mathrm{Z}$

14: Allocation result Update $\mathrm{W}_{\mathrm{i}}=\left[\mathrm{Z}^{*}, \mathcal{X}_{3}\right], \mathrm{i}=\mathrm{i}+1$;

15: end

16: return $\mathrm{W}=\mathrm{W}_{\mathrm{i}}$

According to [11], the sequence $\left\{\Psi\left(\mathrm{W}_{\mathrm{i}}\right)\right\}$ is non-decreasing after each iteration and converges to a stationary solution of problem (10), when $\mathrm{W}_{1}=\mathrm{W}_{2}=\mathrm{W}_{3}$. Thus the sequence of iteration generated by the IHM converges to at least a local optimal point of the problem (10).

\section{SIMULATION RESULT}

In this section, we compare the performance of the proposed Iterative Hungarian algorithm with greedy algorithm [12], optimal scheme[13], two-step two-side Hungarian algorithm and full-duplex based resource allocation[10].

In greedy algorithm, each $\mathrm{CH}$ select TU-RU pairs with the best security capacity for itself sequentially and exclusively. Note that $\mathrm{CH}$ can also select $\mathrm{TU}$ or RU according to security capacity maximization criterion.

Optimal solution of 3-dimentional matching need exhaustive search for each TU-RU-CH triplet, and the computational complexity is $\mathcal{O}(K ! K !)$ according to [13]. To reduce the complexity, we used a low complexity optimal algorithm for comparing which is implemented in two steps. In the first step, each TU-RU possible pair is obtained by exhaustively searching, which complexity is $\mathcal{O}(K !)$. In the second step, the optimal matching between each TU-RU pair and RUs is accomplished by Hungarian method with complexity $\mathcal{O}\left(\mathrm{K}^{3}\right)$. The computational complexity for this low-complexity optimal algorithm is $\mathcal{O}\left(\mathrm{K}^{3} \mathrm{~K} !\right)[11]$.

In two-step two-side Hungarian method, the 3-dimentional matching problem (TU-RU-CH) is decoupled as two 2dimentional matching problems (TU-CH and RU-CH). Each 2dimentional matching problem is solved by Hungarian method with complexity $\mathcal{O}\left(\mathrm{K}^{3}\right)$. Thus the complexity of TSH is $\mathcal{O}\left(2 \mathrm{~K}^{3}\right)$. Note that the two-step two-side Hungarian method ignores the peer effect between different TU-RU pairs.

We also perform a half-duplex resource allocation in which a $\mathrm{CH}$ can only allocated to a TU or a RU. The simulation parameters are list in table II.

Table II: Simulation Parameters

\begin{tabular}{ll}
\hline Simulation Parameters & Value \\
\hline Pathloss factor & 4 \\
$\begin{array}{l}\text { Distance between any user to BS } \\
\text { Distance between eavesdropper }\end{array}$ & $10-100 \mathrm{~m}$ \\
to BS & $80 \mathrm{~m}$ \\
Interference cancellation & $-60 \mathrm{~dB}$ \\
coefficient & \\
Uplink transmit power & $25 \mathrm{dBm}$
\end{tabular}

Downlink transmit power $\quad 30 \mathrm{dBm}$

Noise Power

$-114 \mathrm{dBm}$

Fig.3 shows the total security capacity as a function of the number of TUs. Fig.4 shows the total security capacity as a function of the number of TU/RU where the number of TU and

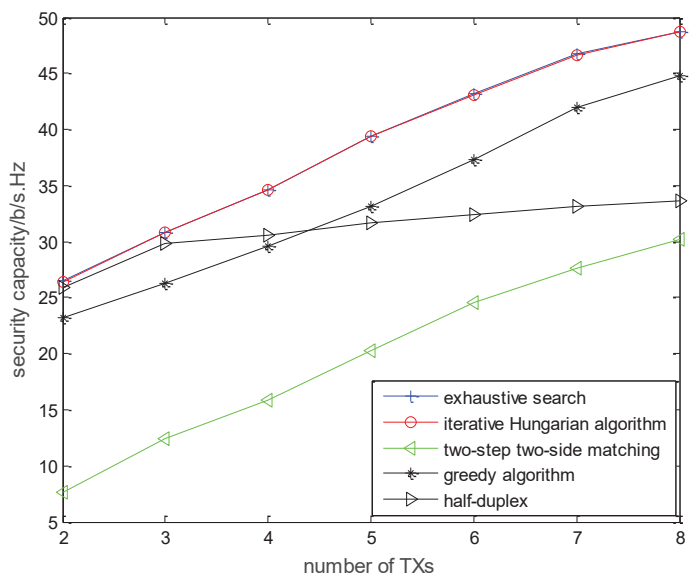

Fig3. Total security capacity vs. number of TUs with 5 RUs and $8 \mathrm{CHs}$

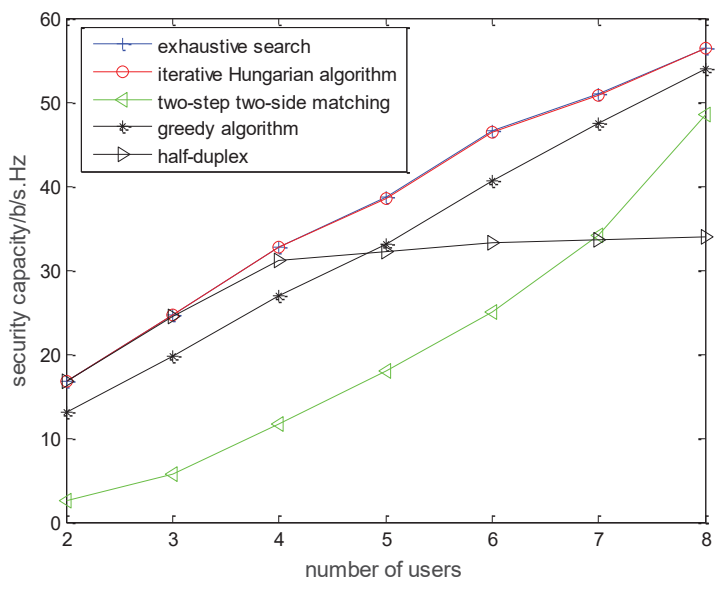

Fig.4 total security capacity vs. number of TU and RU $(\mathrm{M}=\mathrm{N})$ with $8 \mathrm{CHs}$ 


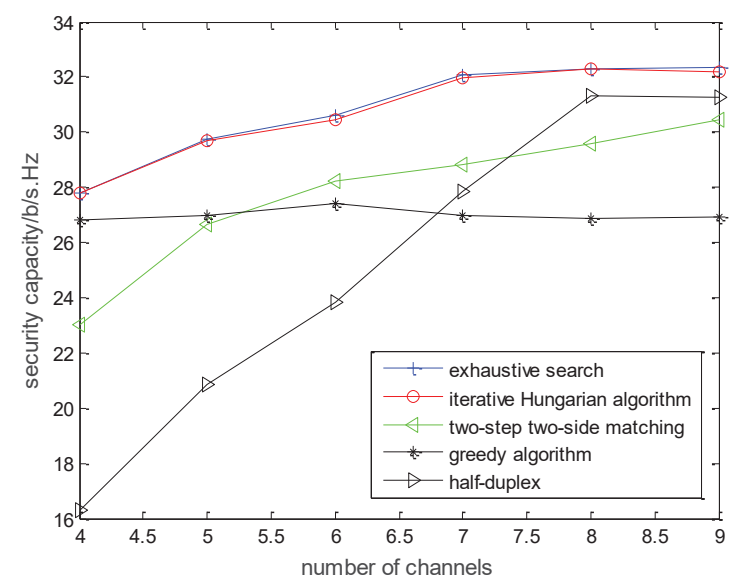

Fig. 5 total security capacity vs. number of channels with 4 TUs and 4 RUs

RU are equal. We can observe that for all algorithms, the total capacity grows as number of TU increases. Also, the proposed iterative Hungarian algorithm can nearly achieve the performance of exhaustive search and outperform greedy algorithm, two-step two-side matching algorithm, half-duplex resource allocation significantly. When $\mathrm{M}+\mathrm{N}<\mathrm{K}$, the performance of half-duplex resource allocation is slightly worst than iterative Hungarian algorithm which is based on full-duplex mode. When $\mathrm{M}+\mathrm{N}>\mathrm{K}$, for half-duplex resource allocation, the number of channels is not enough to serve all TUs and RUs due to TUs and RUs are assigned with different channels. In this case, the gap between half-duplex resource allocation algorithm and iterative Hungarian method become significantly larger. In twostep two-side matching, the matching of TU-CH and RU-CH do not consider the peer effect between RU and TU, thus the performance is much worse than iterative Hungarian method and greedy algorithm.

Fig. 5 shows the total security capacity as a function of number of channels with 4 uplink users and 4 downlink users. The exhaustive searching and iterative Hungarian algorithm achieve the best performance comparing other three algorithms. The performance of half-duplex based resource allocation increase rapidly with the number of channels increasing, when the number of channels is less than summation of number of TU and number of $\mathrm{RU}(\mathrm{K}<\mathrm{M}+\mathrm{N})$, because in this case, more users (TU or $\mathrm{RU})$ can access wireless channel with number of channels increasing. When the channel is enough for all TU and RU $(\mathrm{K}>\mathrm{M}+\mathrm{N})$, the increasing of channel brings less benefit. the performance of greedy algorithm is almost constant. Every channel select the best TU-RU pair for itself sequentially in greedy algorithm and each TU or RU only can be selected once due to fairness consideration. When the number of channels is larger than that of TU/RU, all TU/RU are selected by the forward channels $(\mathrm{CH} 1, \ldots \mathrm{CHM})$ and the left channel can only choose virtual TU and virtual RU.

\section{CONCLUSIONS}

We investigated resource allocation for full-duplex cellular system to improve physical layer security. Through a 3dimentional matching approach, an iterative Hungarian algorithm is proposed to solve the proposed resource allocation problem. In each iteration, a 2-dimentional matching of one set and the matched pairs in other two set is performed. We analyzed the complexity and several other algorithms. Simulation results show that IHM can nearly achieve optimum solution with a significantly lower complexity.

\section{ACKNOWLEDGMENTS}

This work is supported by Fundamental Research Funds for Central Universities under Grand (2014QNB46), National Nature Science Foundation of China (No.51504255, No.51504214, No.51274202), The Fundamental Research and Development Foundation of Jiangsu Province (No.BE2015040).

\section{REFERENCES}

[1] D. Kim, H. Lee, D. Hong, A survey of in-band full-duplex transmission: From the perspective of PHY and MAC layers[J]. IEEE Communications Surveys \& Tutorials, 2015, 17(4): 2017-2046.

[2] A. Thangaraj, R. K. Ganti, and S. Bhashyam, "Self-interference cancellation models for full-duplex wireless communications," in Proc. Int. Conf. Signal Process. Commun. (SPCOM), Bangalore, India, Jul. 2012, pp. 1-5.

[3] A. Wyner, The wire-tap channel, Bell Sys. Tech. J., vol. 54, no. 87, 1975.10, pp. 1355-87.

[4] W. Trappe, The challenges facing physical layer security[J]. IEEE Communications Magazine, 2015, 53(6): 16-20.

[5] L. J. Rodriguez, N. H. Tran, T. Q. Duong, et al. Physical layer security in wireless cooperative relay networks: State of the art and beyond[J]. IEEE Communications Magazine, 2015, 53(12): 32-39.

[6] Z. Qin, Y. Liu, Z. Ding, et al. Physical layer security for $5 G$ non-orthogonal multiple access in large-scale networks[C]//Communications (ICC), 2016 IEEE International Conference on. IEEE, 2016: 1-6.

[7] W. Wang, K. C. Teh, K. H. Li, Enhanced Physical Layer Security in D2D Spectrum Sharing Networks[J]. IEEE Wireless Communications Letters, 2016.

[8] F. Zhu, F. Gao, T. Zhang, et al. Physical-layer security for full duplex communications with self-interference mitigation[J]. IEEE Transactions on Wireless Communications, 2016, 15(1): 329-340.

[9] F. Zhu, F. Gao, M. Yao, et al. Joint information-and jamming-beamforming for physical layer security with full duplex base station[J]. IEEE Transactions on Signal Processing, 2014, 62(24): 6391-6401.

[10] B. Di, L. Song, Y. Li, H. Zhu, Joint User Pairing, Subchannel, and Power Allocation in Full-Duplex Multi-User OFDMA Networks, IEEE Trans. Wireless Commun., vol.15, no.12, pp.8260-8272.

[11] T. Kim, M. Dong, An iterative Hungarian method to joint relay selection and resource allocation for D2D communications[J]. IEEE Wireless Communications Letters, 2014, 3(6): 625-628.

[12] G. Li, H. Liu, Resource allocation for OFDMA relay networks with fairness constraint, IEEE J. Sel. Areas Commun., vol. 24, no. 11, pp. 2061-2069.

[13] Z. Lu, Y. Shi, W. Wu, B. Fu, Efficient data retrieval scheduling for multichannel wireless data broadcast, IEEE Infocom, 2012, pp. 891-899. 